\title{
Un estudio comparado del valor económico y social de dos universi- dades adventistas
}

\section{A comparative study of the economic and social value of two Ad- ventist universities}

\author{
Gustavo Gregorutti*1, Olandy Naranjo Rivera², Carlos William Marín ${ }^{3}$ \\ ${ }^{1}$ Facultad de Educación, Andrews University \\ ${ }^{2}$ Facultad de Ciencias Administrativas y Contables, Corporación Universitaria Adventista de Colombia \\ ${ }^{3}$ Dirección de Investigación, Corporación Universitaria Adventista de Colombia
}

\section{INFORMACIÓN DEL ARTÍCULO}

Historia del artículo:

Recibido el 22 de marzo de 2015

Aceptado el 29 de mayo de 2015

\section{Palabras clave:}

Aporte social

Universidades emprendedoras

Financiamiento para la educación

Industrias educativas

\section{Resumen}

Este estudio busca entender los factores centrales que contribuyen a la continuidad exitosa de las industrias alimenticias de dos universidades adventistas en Colombia y Perú. Dichas fábricas han estado contribuyendo social y económicamente a través de oportunidades de empleo para estudiantes de estratos sociales bajos. La investigación recogió datos de una serie de entrevistas a gerentes de ambas fábricas. Los resultados evidenciaron que el éxito de continuidad y aporte está relacionado con relaciones administrativas eficientes entre universidad y fábricas y una cultura de innovación que busca tener los mejores recursos humanos para adaptarse a los mercados. Los entrevistados también expresaron algunas preocupaciones para el desempeño futuro del actual modelo de universidad-industria. El trabajo concluye con una discusión general y algunas sugerencias para el avance futuro de estas empresas y sus aportes.

\begin{abstract}
Abstrac
This study seeks to understand the key factors that contribute to the successful continuation of the food industries of two Adventist universities in Colombia and Peru. These factories have been contributing socially and economically through employment opportunities for students from lower social strata. The study gathered data from a series of interviews with managers of both factories. The results showed that the success of continuity and contribution is related to efficient administrative relations between universities and factories and a culture of innovation that seeks to have the best human resources to adapt to markets. Respondents also expressed some concerns for the future performance of the current model of university-industry. The paper concludes with a general discussion and some suggestions for the future progress of these companies and their contributions.
\end{abstract}

\footnotetext{
* Autor de correspondencia: Andrews University, 4195 Administration Drive, Berrien Springs, 49104, Michigan, EEUU

Correo electrónico: ggregoru@andrews.edu, teléfono: +1-269-471-6163
} 


\section{Introducción}

En las últimas décadas, la universidad privada ha estado contribuyendo notoriamente en la creciente masificación de la educación superior en el Caribe y Latinoamérica (Salmi, 2007). Pero al mismo tiempo, eso significa que estudiar conlleva un creciente costo reavivando la problemática de la igualdad de oportunidades para importantes grupos sociales que necesitan de un título universitario como medio directo de integración a una economía cada vez más estimulada por la especialización y el conocimiento (Toakley, 2004).

Las universidades privadas han estado experimentando diversas modalidades para sostener sus operaciones, pero es evidente que la forma en que la educación es financiada está relacionada con la idea de universidad y sus funciones o misiones primarias (Boyer, 1997; Scott, 2006). El mecanismo más simple es cobrar matrículas y cuotas basadas en la enseñanza para mantener la mayor parte de sus estructuras y personal. Pero la dificultad está en que esos ingresos no cubren la mayoría de los costosos requerimientos de la educación superior, incluyendo a aquellos estudiantes que solicitan más ayuda financiera (Archibald y Feldman, 2010; Erdman, 2008). Algunas instituciones han logrado incorporar nuevas formas de ingresos a través de la creación de servicios $y$, particularmente, conocimiento (Slaughter \& Rhoades, 2004). La misión de impactar la sociedad a través de invenciones para crear riqueza y empleo predomina en muchas universidades que lideran rankings y son muy influyentes (Clark, 2001; Rauhvargers, 2011). Esto ha creado el negocio de la investigación como fuente de entradas para financiar proyectos, becas de estudio e transferencia de invenciones (Gaffikin y Perry, 2009). En consecuencia, la comercialización de la investigación se promueve a través de las alianzas entre universidad-industria-gobierno, que generan dinero de patentes y licencias. Este modelo de universidad trae una cantidad importante de recursos a las instituciones que son capaces de competir estratégicamente bajo las condiciones requeridas para descubrir ideas.

El problema es que un modelo de investigación-financiación es factible, sobre todo, para un grupo selecto de instituciones que están alineadas con la investigación como una función central. Aunque eso no es un problema en sí mismo, la mayoría de las universidades privadas están lejos de adoptar un modelo tal (Gregorutti, 2012). Entonces, si el sistema actual como alternativa de financiamiento externo es difícil de lograr, ¿qué otra opción hay, además de la matrícula y la investigación? Particularmente en el caso de la educación adventista, y desde un comienzo, se practicaron múltiples modos de ingresos hacia un modelo de sostenimiento centrado en el autofinanciamiento del alumno, como lo destaca Elena de White (2001), "En todo lugar donde se establezcan escuelas debemos estudiar qué industrias, que provean empleo a los estudiantes, se pueden empezar" (p. 579). La idea de desarrollar industrias e ingresos paralelos, para que los estudiantes se paguen sus costos, fue medular en la creación de otras instituciones terciarias en países de bajos recursos. Además, en los orígenes de este modelo sostenimiento de los estudiante había un doble propósito, como Elena de White (1913/1991) lo expresa en la siguiente cita:

Por otro lado, ¡cuánto podría ganarse si se siguiese el plan del sostén propio! El estudiante se vería a menudo capacitado para dejar la institución educativa casi o completamente libre 
de toda deuda personal; las finanzas de la escuela estarían en condición más próspera; y las lecciones aprendidas por el estudiante mientras adquiría esta experiencia en su propio campo, le serían de valor indecible en los campos extranjeros. (p. 513)

Basadas en este estándar, muchas instituciones fueron capaces de ofrecer una educación asequible a familias de bajos ingresos. A pesar de que hay diferencias históricas, económicas y sociales, todavía hoy hay instituciones que emplean esos principios para guiar y dar ayuda financiera a sus estudiantes en algunos países de Latinoamérica. Esto es especialmente relevante en el contexto de los colegios y universidades que han estado cerrando industrias desde hace unas tres décadas, haciendo difícil el acceso de una educación universitaria a jóvenes pobres (Gregorutti, 2012).

Dada la relevancia de la contribución educativa de una universidad, este artículo se enfoca en dos universidades adventistas de Latinoamérica que han desarrollado y mantenido industrias por muchos años haciendo aportes sociales y económicos importantes. A continuación se presentan sus características, estrategias y desafíos para brindar oportunidades a jóvenes de diversos estratos sociales. Estos casos pueden servir de extrapolación para otras instituciones que están buscando enriquecer sus aportes.

\section{Marco teórico}

En el contexto de la problemática planteada anteriormente, es oportuno describir inicialmente el concepto de empresa social para contextualizar el modelo de las universidades adventistas, cimentado en el desarrollo de un objeto social que busca favorecer a los estudiantes de bajos recursos a través de mecanismos de financiamiento alternativos.

La empresa social y sus contribuciones

Al inicio de los 90s comenzó a delinearse un nuevo modelo de empresa social para las organizaciones que buscan ir más allá del lucro. Duque (2007) aclara precisando que son aquellas empresas "cuya finalidad es la solución de los problemas sociales básicos de los seres humanos de una manera autosuficiente y rentable..." (p. 61). Es decir que por medio de negocios o emprendimientos buscan impactar mediante cambios sociales como característica primordial de sus misiones institucionales (Nicholls, 2006). Esto es posible a través de una estrategia productiva que genere rentabilidad y sostenibilidad a largo plazo. Así, la empresa social se entiende como una estrategia productiva cuyo ámbito de elección es la asistencia y la redistribución de bienes de modo rentable para sus promotores (De Leonardis, Mauri, Rotelli, \& Eguía, 1995).

Respecto a la visión de sostenibilidad y rentabilidad, Haugh (2005) señala que en la empresa social se fusionan los recursos y capacidades corporativas con el fin de contribuir al mejoramiento social, pero dentro de una lógica de un modelo de negocio que comercializa bienes y servicios auto sostenibles en el tiempo (Vázquez-Maguirre \& Portales, 2014). Sin embargo, también existen propuestas de empresa social en las cuales el modelo de negocio no se encuentra fundamentado exclusivamente a partir de la venta de productos o servicios como mecanismo único de financiamiento, sino que también incluyen otras fuentes de ingresos provenientes de donaciones en efectivo o en especies, asignaciones de dinero públicos nacionales o extranjeros, o partidas presupuestarias internas de una organización que no es parte de la gestión 
de la empresa social beneficiada (Casasnovas \& Vernis, 2011).

Por lo mencionado, es importante entender las distintas formas en que se obtienen y producen recursos con fines sociales. En el sector privado, y en el caso de las universidades adventistas, las instituciones operan a través de mecanismos de financiamiento de mercado (Austin, Gutierrez, Ogliastri, \& Reficco, 2006)Gutierrez, Ogliastri, \&amp; Reficco, 2006, pero con objetos sociales para subsidiar, por ejemplo, el costo de matrículas y manutención de estudiantes durante el desarrollo de la vida académica en la universidad. El mercado opera en este caso como el medio para desarrollar el intercambio, y los clientes y/o consumidores deben estar dispuestos a pagar el precio de los bienes y servicios ofrecidos por la empresa social para generar el valor económico de la organización (Rosselló \& Salvà, 2011). De acuerdo a Austin et al., (2006) dicho valor se entiende "como aquel cuyos beneficios pueden ser capturados y remunerados libremente por sus receptores, con un precio que excede su costo de producción" (p. 286).

La verdadera promesa de una empresa social, así como su futuro, está en su potencial de ser una metodología para la organización sin fines de lucro que busca el cumplimiento de una misión y el fortalecimiento en su capacidad para lograr un impacto social sostenible a través de sus estrategias innovadoras. La riqueza del emprendimiento social se encuentra en que el trayecto de las prácticas de negocios se pueden entrecruzar con una mejora social y ofrecer más contribuciones que generar dinero. Estos nuevos paradigmas deben enfatizar la misión como la piedra angular de la empresa social para que se centren en los modelos operativos que maximicen su impacto (Nasruddin \& Fahada Misaridin, 2014). Establecen así un doble valor: el social y económico (Alter, 2006). Desde esa perspectiva, la razón de ser de una organización sin fines de lucro es el cambio social que motiva su devenir diario; la obtención de dinero es sólo algo secundario ya que se lo entiende como un medio necesario para la financiación de los fines humanitarios (Nicolás Martínez \& Rubio Bañón, 2012).

Por lo tanto, a partir de esta conceptualización, las instituciones adventistas generan un valor social representativo en la medida que intentan derribar barreras de acceso a la educación para jóvenes que se encuentran en condiciones económicas desfavorables.

\section{Materiales y método}

Este estudio utiliza una metodología de casos, cualitativa y comparada. Se basa en el análisis de los modelos de industria que han implementado la Universidad Adventista de Colombia y en la Universidad Peruana Unión, en Perú. La pregunta central que dirige este trabajo es: ¿qué clase de modelo de industria han desarrollado para auxiliar financieramente la educación de sus alumnos a través de empleo? Además, el estudio provee información de las características, contribuciones y desafíos de estas dos universidades adventistas. Dichas instituciones han sido capaces de mantener fábricas de alimentos con un posicionamiento importante en el mercado y rédito para asistir activamente a estudiantes necesitados. Esta contribución social y económica es relevante para sus respectivas regiones, países y la Iglesia Adventista que las patrocina.

Las dos universidades fueron seleccionadas por poseer industrias con décadas de trayectoria y aportes a las economías locales y regionales sirviendo, a lo largo de los años, a miles de estudiantes de 
estratos sociales bajos. Es importante destacar que la mayoría de las universidades adventistas en Latinoamérica han cerrado sus fábricas convirtiendo a estas dos instituciones en casos de estudio que representan continuidad y éxito gerencial. Se dejó de lado otras alternativas de financiamiento que estas y otras universidades ponen en práctica para ayudar, tales como la venta de libros y empleos dentro de los campus durante los veranos y el año escolar.

Como ya se expresó, este trabajo usa una metodología cualitativa de casos comparados para entender las características de los procesos implementados. Esta técnica es la más apropiada para su análisis (Creswell, 2009; Holliday, 2007). Para tal efecto, se procedió a la recolección de datos a través de entrevistas. En la Universidad Peruana Unión se entrevistó al gerente general de la universidad y se llevó a cabo un grupo de enfoque con tres gerentes de la fábrica de alimentos Productos Unión. Uno de ellos era el gerente general y los otros dos de las áreas comercial y de marketing. Para la Universidad Adventista de Colombia, se condujeron dos entrevistas individuales con el gerente general y el vicegerente de la planta de productos Vitarrico. Se seleccionó a los administradores porque ellos son los más influyentes en la toma de decisiones haciéndolos aptos para responder a las preguntas de los investigadores.

Las entrevistas tuvieron una extensión de unos 30 minutos de promedio. La primera pregunta con que se comenzó fue, ¿por qué cree que algunas fábricas están siendo cerradas y otras son capaces de sobrevivir y crecer aun cuando dependen de instituciones académicas? A medida que se avanzaba en las entrevistas, se agregaron nuevas preguntas para expandir nuevas ideas. Luego todas estas informaciones fueron transcriptas y pos- teriormente analizadas para detectar los temas emergentes que surgieron de las respuestas. Los resultados son expuestos en la siguiente sección.

\section{Resultados}

De las entrevistas emergieron tres grandes tópicos, a saber, el modelo que se implementa en cada caso, cómo se contribuye en lo financiero, educativo y social, y finalmente los desafíos que estas dos industrias enfrentan para mantenerse y crecer en el contexto de dependencia administrativa. A continuación se presentan los resultados entorno a esos tópicos y subtemas que emergieron en las entrevistas de cada una de las dos universidades.

\section{Modelo de empresa social}

\section{Características administrativas}

Un primer punto preponderante de las entrevistas es que las industrias parecen verse afectadas por el tipo de contabilidad que usan para administrarlas, como asegura este gerente en Perú, "Es fundamental un sinceramiento de los costos reales de la industria y los que corresponden a la universidad para que se puedan medir en realidad si la gestión industrial está siendo bien llevada o no." El mismo administrador se pregunta: "¿Cómo vemos a la industria, como una caja chica para sacarle dinero?" El gerente general de la fábrica de Colombia resume la misma idea de la siguiente manera, "... podemos decir que el problema es netamente de gestión administrativa." Sin embargo, estas instituciones tienen un claro sentido misional que las alínea con las necesidades y propósitos de sus universidades, como expresa el gerente de Colombia, "Nuestra misión es apoyar y dar oportunidades a los estudiantes que no las tienen." En la universidad peruana la integración es expresada en sus 
múltiples modos, "Por un lado la industria quiere crecer como industria, pero a la vez tenemos como objetivo ayudar a los estudiantes, nuestra misión dice que nosotros existimos para apoyar la labor educativa dentro de la universidad." Esta clara relación de propósitos parece facilitar un desarrollo conjunto de las partes involucradas.

Otra variable que parece ser central para la industria en Colombia es que, como expresa el gerente, "En esta industria existe una estrecha relación y es así como debería ser en todas las universidades...no puede trabajar de otra manera." La estructuras siguen la siguiente modalidad, "Nosotros como industria tenemos nuestra administración...Cuando se trata de votos de mayor envergadura, esa junta lo recomienda a la junta de dirección de la universidad y así a la Unión si es mayor." Un modelo muy similar implementa Perú. O sea que la industrias siguen la típica trayectoria de administración eclesiástica.

\section{Empleados}

Otro elemento central para un buen funcionamiento está dado en la forma cómo se incorpora la mano de obra de los estudiantes. En el caso peruano, se emplean jóvenes que tienen disponibilidad de más horas semanales porque estudian los domingos solamente, "...el tipo de estudiantes que tenemos, son los que estudian los domingos, los que estudian durante la semana es muy complicado asignarles una tarea de 8 horas, tenemos aproximadamente 50 estudiantes que están ejerciendo así." Estos cumplen funciones rutinarias y son parte del plantel de empleados. En el caso de Colombia, la dependencia de mano de obra de estudiantes es de un $40 \%$ de estudiantes durante el año escolar, y contratan alumnos que la universidad les destina, "...es- tamos limitados a los planes disponibles y no podemos nosotros crear otro tipo de plan adicional para no crear un desbalance o desigualdad con los otros departamentos o las otra industrias que existen dentro de la universidad." Esto indica una estrecha y coordinada colaboración con la universidad.

En el caso peruano, existe la variante de venta domiciliaria, "Los domingos algunos alumnos se organizan para salir a vender pan en grupo con un carro de aquí, de la UPeU. Incluso hay un grupo que vende pan, granola, galletas, cocada, entre otras cosas, fuera del país." Esto permite que los jóvenes se mantengan financieramente y representen acerca de un $10 \%$ de las ventas anuales de la fábrica. Este modelo de venta puerta por puerta es conveniente para los estudiantes y para la universidad porque alrededor de un $20 \%$ del alumnado lo practica.

Además, de acuerdo al gerente general peruano, el personal especializado y capacitado es una necesidad constante, para tal fin se implementa un sistema de perfeccionamiento continuo para ayudar en la deficiencias de entrenamiento, "Una de las prácticas que tenemos aquí es que en nuestro personal gerencial tenemos un plan de permanente actualización."

\section{Valor social y económico de las universi- dades adventistas}

Las contribuciones de las industrias estudiadas fue el segundo tópico que emergió de las entrevistas. Estas se concentraron en los aportes sociales y económicos que se logran a través de las múltiples actividades.

\section{Valor social}

Como ya se mencionó, el caso de la universidad de Perú representa una estrategia 
de innovación única en Latinoamérica. Una de las modalidades de venta está centrada en estudiantes que ofrecen pan y otros productos puerta a puerta. Esto no sólo trae beneficios monetarios a los alumnos, sino que también promueve la Iglesia y sus instituciones, como expresa este gerente:

Este segmento de venta puerta por puerta es significativo porque cuando ese joven toca la puerta de una casa, la gente dice no solo estoy consumiendo un producto saludable, sino que también siente que está ayudando a un joven a que estudie y allí hay un impacto en el público. Además, la Iglesia es conocida a través de sus instituciones.

Esta metodología posibilita que haya múltiples beneficiados agregando valor social a la actividad. La Universidad Peruana Unión logra una promoción de su marca educativa sin costos adicionales. Esto agiliza el reclutamiento natural de los jóvenes de menos ingresos que ven en este tipo de venta una opción para pagar sus estudios.

Por otra parte, la gerencia de las industrias y universidades se esfuerzan por crear productos innovadores. Esto se aplica a ambas universidades que buscan entrar al mercado con algunos alimentos que son novedosos, como asegura este gerente de producción,

Tenemos que de alguna manera darle valor agregado a nuestros productos porque ese es nuestro sello. Queremos crecer y creemos que podemos dar más y obviamente empezamos a sacar líneas de cosas dulces sin alcohol, es un mercado cada vez más demandante, porque hoy hay una variedad creciente de cereales orgánicos, hay gente que los compra. Por ejemplo la universidad tiene más de 52 productos por lo menos aquí y tenemos 13 líneas de producción.
Estas respuestas dan evidencias de continuas estrategias para expandir servicios y productos que el mercado puede absorber. Ese tipo de enfoque es proactivo buscando el lado innovador como camino para el crecimiento.

\section{Valor económico}

Estas instituciones atienden un grupo importante de alumnos con necesidades financieras, como lo destaca este gerente de Colombia,

El impacto social es grande porque toda esa cantidad de jóvenes que no tiene ninguna posibilidad se vienen aquí buscando oportunidades de ser profesionales, que es un gran impacto para la sociedad, vemos que no lo existe en otra institución, en otra industria, en otra universidad y para nosotros es reconfortante escuchar de boca de personas de afuera que llegan acá y quedan maravillados con el plan que les presentamos. Aunque nos resulta caro tener estudiantes porque podríamos desarrollarnos con tecnología y poner toda una planta automática con 3 o 4 personas, pero sentimos que nos estaríamos desviando del propósito por el que esta industria está aquí.

Es evidente que esos estudiantes deben estar dispuestos a trabajar para solventarse, pero si lo desean, la universidad les da una oportunidad y al mismo tiempo generan ingresos para la industria que hace esfuerzos por brindarles soluciones. Esto es diferente de darles reducciones o becas cortando los presupuestos internos o global institucional.

Según el gerente de la universidad de Perú, el sistema de trabajo por estudio aporta a todas las carreras, pero especialmente lo hace en mayor medida en las humanísticas, 
Notamos que los alumnos que tienen más dificultad económica son los de educación, teología y un poco de psicología, por alguna razón son los que más salen a colportar (vender libros) y a vender productos alimenticios, el resto de carreras tienen mejor sostenimiento personal cada uno por su parte.

Ese grupo de estudiantes parece estar en mayor desventaja que el resto y la universidad les brinda una alternativa para conseguir un título profesional. Estos intercambios se insertan en el contexto del programa de Beca 18 o Modalidad Formativa Laboral que el gobierno destina para estudiantes de bajos recursos. Así se reciben estudiantes becados por el gobierno ofreciéndoseles también opciones laborales en las industrias con programas adaptados a sus tiempos y necesidades.

Finalmente, las industrias comparten sus utilidades representando una importante fuente de ingresos para el presupuesto general de las universidades, a parte de las becas de estudio que se generan a través del trabajo.

\section{Desafíos}

Como toda organización, estas industrias enfrentan algunos desafíos que se relacionan con sus modelos de industria. Es decir, ¿qué expectativas se tienen para la producción y expansión? Esto está íntimamente relacionado con el nivel del capital humano disponible, como el siguiente gerente de Perú clarifica esa tensión:

La falta de capacitación técnica del estudiante y de su continuidad nos pone un reto. Para eso pensamos que con la ayuda de la tecnología podríamos tener estudiantes como auxiliares de producción, para que el impacto de su participación no ponga en riesgo nuestra producción... llegamos a un punto en en el cual si queremos competir con economía de escala, tenemos que automatizar la empresa.

El problema de recursos humanos aptos para administrar la empresa es también evidente en ambas fábricas. Esto no solo se da con los alumnos, pero también con la gerencia, según este gerente de Colombia, "...en la Iglesia Adventista, dentro de la nuestras estructuras organizacionales, no se forman empresarios y no es lo mismo la visión de un académico que la de un empresario." Además, los planes de crecimiento pueden significar un desafío importante para el modelo de integración estudiantil en la fábrica, como el mismo gerente reconoce, "pero si el estudiante sigue participando en el trabajo menos calificado, será difícil que pueda realmente pagar sus estudios." En el caso de Colombia, uno de los gerentes entiende la tensión de la siguiente manera,

...estas industrias se crearon dentro de un sistema económico proteccionista y ahora estamos abriéndonos al mercado para que vengan otro tipo de organizaciones y nosotros no nos hemos preparado para esos cambios. Claro nos golpean con productos más innovadores, con costos muy bajos y nuestras industrias no han logrado posicionarse muy bien en costos y en diferenciación de productos.

Esa tensión se encuentra a la base del sistema de industrias asociadas a universidades, haciendo el balance difícil y complejo para darles futuro, como lo dice este vicegerente de Colombia, "Cuando tienes en una misma figura administrativa dos unidades de negocio y dos tipos de empresa (la educativa y la de industria), a veces descuidas una o la otra porque las dos tienen intereses que pueden ser 
contrapuestos..." Ambas tienen funciones específicas pero deben combinarse porque comparten la misión de ayudar a estudiantes sin arruinarse financieramente. Las toma de decisiones técnicas puede ser también un desafío para la operación exitosa, como asegura este gerente peruano:

Aquí debemos rayar la cancha, porque mientras estemos bajo el paraguas de la universidad necesitamos asegurarnos que las decisiones técnicas se tomen en la planta. Eso conlleva una visión distinta de industria. Lo que me parece que está pasando es que muchas universidades de la Iglesia no le dan la oportunidad de ser realmente una industria y que compita con las de afuera.

Esta combinación de factores hace que el crecimiento de la industria en el contexto de la universidad sea algo conflictivo. El problema se incrementa con la competencia externa que pone presiones de administración, recursos humanos y tecnológicos para ganar mercado.

\section{Discusión}

Volviendo a la pregunta que guía esta investigación, ¿Qué clase de modelo de industria han desarrollado las universidades estudiadas para auxiliar financieramente la educación de sus alumnos a través de empleo? De acuerdo a las entrevistas, estas empresas tienen por lo menos las siguientes características:

\section{Sistema administrativo.}

Poseen un sistema de administración que deja espacio para que los dirigentes y empleados innoven y reaccionando rápidamente a los cambios de mercado. Las estructuras de decisiones no dependen exclusivamente de la administración central de la universidad y las ganancias no son absorbidas totalmente por la universidad. El superávit es distribuido después de haber renovado y mantenido las máquinas y equipos de la producción.

\section{Empleados.}

El modo cómo administran los recursos humanos es otro elemento clave común a estas fábricas que operan con saldos positivos. Se ven muy pocas duplicaciones de estructuras de personal, cantidades innecesarias de alumnos contratados y obreros de tiempo completo para hacer tareas que un empleado más económico puede hacer. Buscan capacitar a su gente y promover eficiencia con directivos entendidos en el negocio.

\section{Cultura emprendedora.}

Finalmente, los negocios prósperos en estas universidades adventistas parecen promover y tolerar una cultura de riesgo hacia la creación de alternativas. Aun cuando no siempre se trabaja en situaciones ideales, se estimulan estrategias innovadoras para competir y crecer.

Si bien estas industrias han sido capaces de brindar un gran apoyo financiero y estratégico a los alumnos, universidades y la Iglesia, su contribución se puede ver seriamente afectada por las tensiones de mercados globalizados con flujo de capitales que incrementan la competencia. Los administradores de ambas industria expresaron preocupación por la competencia. El problema es que el modelo de relación industria-universidad parece tener ciertos condicionamientos de flexibilidad que limitan el crecimiento. Las industrias tienen sus propios desafíos y tiempos que no siempre son entendidos sistémicamente por las estructuras académicas o eclesiásticas. Concretamente, ¿cómo manejar la rotación de administradores que no siempre son los más entendidos 
en la materia?, ¿cómo agilizar la toma de decisiones cuando hay emprendimientos mayores que incluyen personal, como los alumnos, e inversiones millonarias?, ¿hasta dónde la industria debe independizarse de la función educativa para crecer en el mercado? Estas son preguntas que en algún punto deben tratarse porque existe el riesgo de que alguna de los partes involucradas tome decisiones defensivas que impacten las contribuciones que se hacen a los estudiantes de estratos sociales bajos. Dichos conflictos pueden llevar al cierre de las fábricas o a una independencia de la industria muy poco ligada a la función formativa de la universidad. Es un asunto de balance en que por un lado debe haber rédito y por el otro aportes sociales.

\section{Conclusión}

Si bien estas fábricas han sido capaces de aportar social y económicamente a sus universidades, a la Iglesia Adventista y a la sociedad en general, existe el riesgo de una ruptura casi forzada por la creciente demanda especializada y una competencia externa que no perdona. Así, este estudio visualiza algunas de las características básicas que han mantenido a flote estas instituciones, pero también se informan desafíos. Finalmente se propone que las diversas instancias administrativas de la Iglesia, universidades e industrias discutan posibles panoramas de relaciones innovadoras para continuar creando aportes sociales y económicos en sus respectivas esferas de influencia.

\section{Agradecimientos}

Se agradece especialmente a los administradores de las Universidades Adventistas de Perú y de Colombia, y sus respectivas fábricas, por el tiempo y permisos otorgados para recolectar datos a través de entrevistas. 


\section{Referencias}

Alter, S. K. (2006). Social enterprise models and their mission and money relationships. Social entrepreneurship: New models of sustainable social change, 205232.

Archibald, R. \& Feldman, D. (2010). Why does college cost so much? Forbes, August 1. Retrieved from http://www.forbes. com/2010/08/01/rising-cost-education-opinions-best-colleges-10-feldman-archibald.html

Austin, J. E., Gutierrez, R., Ogliastri, E., \& Reficco, E. (2006). Gestión efectiva de emprendimientos sociales: Lecciones extraídas de empresas y organizaciones de la sociedad civil en lberoamérica: Banco Interamericano de Desarrollo.

Boyer, E. (1997). Scholarship reconsidered: Priorities of the professoriate. San Francisco, CA: Jossey-Bass.

Casasnovas, G., \& Vernis, A. (2011). Ecosistemas y emprendimientos sociales. (Spanish). INCAE Business Review, 2(3), 2-6.

Clark, B. (2001). The entrepreneurial university: new foundations for collegiality, autonomy and achievement, Higher Education Management, 13(2), 9-24.

Creswell, J. (2009). Research design. Qualitative, quantitative, and mixed methods approaches (3rd Ed.). Thousand Oaks, CA: Sage

De Leonardis, O., Mauri, D., Rotelli, F., \& Eguía, M. (1995). La empresa social. Argentina: Nueva Visión.

Duque, E. (2007). La empresa social y su responsabilidad social. Revista Innovar, 17(30).
Erdmann, D. (2008). The science of enrollment, the art of admission, the business of financial aid. Independent School, 67(2), 100-108.

Gaffikin, F. \& Perry, D. (2009). Discourses and strategic visions: The U.S. Research University as an institutional manifestation of Neoliberalism in a global era. American Education Research Journal, 46(1), 115-144.

Gregorutti, G. (2012). Alternative models of funding higher education: Past and present trends. In C. Valley, E. Kido, M. Beach, \& D. Herrmann (Eds.), Peril and promises: Adventist education at the crossroads. Riverside, CA: CRAE.

Haugh, H. (2005). A research agenda for social entrepreneurship. Social Enterprise Journal, 1(1), 1-12.

Holliday, A. (2007). Doing and writing qualitative research. Thousand Oaks, CA: Sage.

Mair, J., \& Noboa, E. (2003). Emergence of social enterprises and their place in the new organizational landscape. IESE Working Paper No. D/523. Disponible en http://papers.ssrn.com/sol3/papers. cfm?abstract_id $=462284$

Nasruddin, E., \& Fahada Misaridin, N. A. (2014). Innovation for a social enterprise business model: An analysis of key success factors. International Journal of Organizational Innovation, 7, 149-157.

Nicolás Martínez, C., \& Rubio Bañón, A. M. (2012). El emprendimiento social: una comparativa entre España y países sudamericanos. FAEDPYME International Review, 1(1), 38-49.

Nicholls, A. (2006). Social entrepreneurship: New models of sustainable social chan- 
ge: Oxford University Press.

Rauhvargers, A. (2011). Global university rankings and their impact. Brussels: European University Association.

Rosselló, P. H., \& Salvà, A. S. (2011). Responsabilidad social y contabilidad en las empresas de economía solidaria: estudio teórico y aplicación en Illes Balears. Palma: Institut Balear d'Economia.

Salmi, J. (2007). Autonomy from the state vs. responsiveness to markets. Higher Education Policy, 20, 223-242.

Scott, J. (2006). The mission of the university: Medieval to postmodern transformations. Journal of Higher Education, 77(1), 1-39.

Slaughter, S \& Leslie, L. (2004). Academic capitalism: Politics, policies and the entrepreneurial university. Baltimore: Johns Hopkins University Press.
Toakley, A. (2004). Globalization, sustainable development and universities. Higher Education Policy, 17, 311-324.

Vázquez-Maguirre, M., \& Portales, L. (2014). La empresa social como detonadora de calidad de vida y desarrollo sustentable en comunidades rurales. Pensamiento y Gestión, (37), 255-284.

White, E. (1913/1991). Consejos para los maestros, padres y alumnos acerca de la educación cristiana. Buenos Aires: ACES.

White, E. (2001). El ministerio médico. Miami, FL: Asociación Publicadora Interamericana. 